\title{
Construcción de un índice espacial de bancarización: un estudio para la región centro de Argentina
}

Fernando García

Universidad Nacional de Córdoba

Argentina 


\title{
Construcción de un índice espacial de bancarización: un estudio para la región centro de Argentina
}

\author{
Fernando García ${ }^{1}$ (D) \\ Universidad Nacional de Córdoba-Argentina
}

\begin{abstract}
RESUMEN
La bancarización resulta importante en tanto constituye un motor para el desarrollo económico y social de un país o región al favorecer la disponibilidad de servicios financieros para la población y las empresas y del nivel de acceso y utilización de tales servicios por parte de los distintos agentes económicos. En este sentido, resulta clave una medición adecuada del proceso de bancarización a través de un Índice de Bancarización. Este trabajo propone la construcción de un Índice de Bancarización para la región Centro de Argentina considerando información proporcionada por el Banco Central de la República Argentina y el Instituto Nacional de Estadística y Censos para el año 2010. Como los datos están georeferenciados, resulta adecuado aplicar Componentes Principales Geográficamente Ponderadas que permiten incorporar la heterogeneidad espacial de los datos, es decir considerar situaciones donde los datos espaciales no son bien descriptos por un modelo global. Los resultados permitirían corroborar que esta metodología constituye un aporte metodológico significativo. Se destaca la provincia de Santa Fe, exhibiendo un mayor nivel de bancarización, siguiendo en importancia la provincia de Córdoba, pero con un comportamiento más heterogéneo que contrasta con el de la provincia de Entre Ríos, que aunque presenta un nivel de bancarización menor exhibe un comportamiento más homogéneo.
\end{abstract}

Palabras clave: Bancarización, Componentes Principales Geográficamente Ponderadas.

JEL: G20, G21, R12.

\section{Construction of an Spatial index of banking coverage for the central region of Argentina}

\begin{abstract}
The banking sector is a key sector that enables economic development in any country or region by providing financial services and credit to firms and households. By this, it is crucial to measure how the banking sector evolves in certain regions. This paper constructs a banking index (BI) for the main economic area of Argentina using georeferenced data with information provided by the Central Bank of Argentina and the last Argentinean census. The Geographically Weighted Principal Components Analysis (GWPCA) used seems to be a significant methodological contribution in comparison with the Principal Component Analysis (PCA), since the GWPCA incorporates the spatial heterogeneity ignored by the PCA. As to the results, the BI for the area suggests a higher level of banking coverage for two of the states (Santa Fe and Córdoba). The BI also suggests a heterogeneous behavior in the use the banking services for Córdoba and a homogeneous one for the remaining regional state (Entre Ríos).
\end{abstract}

Keywords: Banking; Geographically Weighted Principal Components. JEL: G20, G21, R12.

1 Departamento de Estadística y Matemática, Instituto de Estadística y Demografía "Dra. Hebe Goldenhersch" y Facultad de Ciencias Económicas de la Universidad Nacional de Córdoba (Argentina) 


\section{INTRODUCCIÓN}

Una mayor bancarización en un territorio implica una mayor disponibilidad, cobertura geográfica, acceso y utilización de los servicios ofrecidos por los bancos y/u otras entidades que forman parte del sistema financiero; circunstancia que propende a un mayor crecimiento económico y favorece a la reducción de los niveles de pobreza (Grupo de Monitoreo Macroeconómico, GMM, 2011; Anastasi et al., 2010).

La bancarización constituye una medida adecuada del grado de desarrollo del sistema financiero de un país, de la disponibilidad de sus servicios para la población y las empresas, y también del nivel de acceso y utilización de los servicios financieros por parte de todos los agentes económicos. En este sentido, resulta indispensable una adecuada medición del nivel y grado de la disponibilidad y utilización de los servicios bancarios en tanto facilita el diseño de medidas de política apropiadas que promuevan una mayor y mejor bancarización (García, 2017).

En los últimos años, la bancarización se ha convertido en un objetivo importante en todos los países y en particular en la Argentina. En esta dirección, se puede mencionar la puesta en marcha del Plan Nacional de Bancarización Inclusiva 2015-2019 por parte del Banco Central de la República Argentina (BCRA), el cual contempla un conjunto de acciones y medidas tendientes a garantizar una mayor inclusión financiera en el país. (García, 2018).

En esta dirección, resulta clave la construcción de un Índice de Bancarización (IB) que permita una adecuada medición de la evolución del proceso de bancarización en tanto contribuye a que exista un reconocimiento respecto a la trascendencia de la bancarización como elemento clave para el crecimiento y desarrollo económico. Despierta particular interés el caso de la Región Centro, integrada por las provincias de Córdoba, Entre Ríos y Santa Fe. ${ }^{2}$ Esta región posee una inmejorable ubicación estratégica para la conexión interna regional, nacional y de integración con los países del Mercado Común del Sur. La región ocupa el segundo lugar luego de la región de Buenos Aires, generando algo más del 20\% del Producto Bruto Geográfico (PBG) de la Argentina, por lo que su economía tiene una importancia clave para el desarrollo socio-económico del país.

En un correlato casi directo con la participación en el PBG, desde el punto de vista de la intermediación financiera, la región es la segunda en importancia en relación a la presencia de infraestructura bancaria. Información provista por el BCRA a diciembre 2019 da cuenta que el número de sucursales bancarias y cajeros automáticos para la región comprende $23,5 \%$ y $22,0 \%$ del total de sucursales y cajeros automáticos existentes en el país respectivamente. Este trabajo propone la construcción de un Índice de Bancarización para la Región Centro de la Argentina para el año 2010, usando información georreferenciada a nivel departamental y aplicando la metodología Análisis de Componentes Principales Geográficamente Ponderadas (GWPCA, son sus siglas en inglés), una extensión del Análisis de Componentes Principales (PCA, son sus siglas en inglés) a datos espaciales.

\footnotetext{
2 La Región Centro se constituye a partir de la suscripción de acuerdos firmados todos en el marco de las facultades otorgadas a las provincias por la Constitución Nacional (Art. 124).
} 
El resto del trabajo se organiza como sigue. En la sección 2 se hace una breve revisión de las principales contribuciones en torno a la bancarización y a las aproximaciones empíricas que tratan de medirla. En la sección 3 se presenta la metodología estadística propuesta para la construcción del IB. La sección 4 presenta la construcción del IB. Finalmente, en la sección 5 se ensayan algunos comentarios finales.

\section{MARCO CONCEPTUAL}

El GMM (2011) considera a la bancarización como "el nivel de disponibilidad, acceso y utilización de la población de un país o una región económica de los servicios ofrecidos por los bancos y/u otras entidades que forman parte del sistema financiero" (p.15). En la misma sintonía, el BCRA (2015) refiere al uso masivo, por parte de los individuos, del sistema financiero formal, relacionando el concepto de bancarización con el acceso, la utilización, la calidad de los servicios financieros y el beneficio que ello genera a la población. Morales y Yáñez (2006) en tanto, en un sentido más amplio, conciben la bancarización como el "establecimiento de relaciones estables y amplias entre las instituciones financieras y sus usuarios respecto de un conjunto de servicios financieros disponibles" (p.9).

Según señala García (2018) "la construcción de un índice de bancarización no ha recibido demasiada atención en la literatura, siendo prácticamente nulos los trabajos para la Argentina" (p.61). Sin embargo, existen antecedentes interesantes respecto a índices de este tipo, destacándose el trabajo de Cámara y Tuesta (2014). Los autores plantean que la medición multidimensional de la inclusión financiera resulta importante en tanto una medida que agrega varios indicadores en un solo índice ayuda a resumir la naturaleza compleja de la inclusión financiera y comprender mejor el fenómeno de interés.

Existen dos enfoques comúnmente utilizados para la construcción de índices compuestos. El primero asigna en forma exógena los pesos relativos de cada indicador sobre el índice global. Dentro de este enfoque se pueden mencionar los trabajos de Sarma (2008, 2012), Arora (2010), Gupte et al. (2012), Chakravarty y Pal (2013), Hasan e Islam (2016) y Sethy (2016) entre otros. El segundo enfoque en cambio, plantea la determinación en forma endógena a partir de la estructura de covariación de los indicadores considerados de los pesos relativos de cada indicador parcial. En esta línea se inscriben los trabajos de Zulaica Piñeyro (2013), Amidzic et al. (2014), Cámara y Tuesta (2014) y Mishra et al. (2014) entre otros. En García (2018) puede encontrarse una revisión detallada de los aportes de estos trabajos. Para Latinoamérica, el antecedente más importante lo constituye el documento del GMM (2011), el cual propone un indicador compuesto de bancarización para los países miembros del MERCOSUR. Aunque interesante, en tanto posibilita una medición del fenómeno de manera global y sintética, dicho indicador no considera la dimensión espacial de los datos; es decir ignora la presencia de patrones espaciales subyacentes en los datos.

Para la Argentina, García (2017) propone la construcción de un índice de bancarización para la región Centro incorporando la dimensión espacial de los datos. Aplica la metodología MULTISPATI propuesta por Dray et al. (2011) siguiendo la práctica común de considerar sólo las primeras componentes en tanto permiten explicar la mayor parte de la variabilidad de los datos. En un trabajo posterior, García (2018), utilizando idéntica 
metodología, sigue la sugerencia de Cámara y Tuesta (2014) de incorporar en el cálculo del indicador todas las componentes ya que el objetivo final no es reducir la dimensionalidad de los datos sino mejorar la precisión en la medición. Bajo estos preceptos, este trabajo propone un IB que introduce en su cálculo la restricción dada por los datos georreferenciados pero, a diferencia de los trabajos anteriores, permite incorporar la heterogeneidad espacial de los datos. En este sentido, la aplicación de GWPCA constituye un aporte metodológico significativo en la construcción del IB.

\section{METODOLOGÍA}

En la construcción del Índice de Bancarización se utilizó el PCA. Domínguez et al. (2011) señalan que el uso de esta metodología se ha generalizado fundamentalmente en situaciones donde no existe un consenso entre los especialistas sobre la importancia relativa de las variables. Adicionalmente, García (2017) señala que la utilización del PCA se justifica por la posibilidad de incorporar datos georeferenciados -es decir asociados con una localización geográfica-, en la construcción de dicho índice constituyendo de esta manera, una propuesta original para la medición del nivel de bancarización. Dray et al. (2011), proponen el método MULTISPATI, una extensión del PCA que incorpora la restricción dada por los datos espaciales mediante el cálculo del índice de Moran (Moran, 1950) para medir la autocorrelación espacial entre las observaciones, para lo cual es necesario definir una matriz de pesos espaciales. ${ }^{3}$

Sin embargo, esta metodología no toma en cuenta la presencia de heterogeneidad espacial, la cual refiere a la naturaleza no estacionaria de los procesos geográficos. Esto significa que los procesos varían localmente y no es necesariamente el mismo en cada localización del espacio geográfico (Demsar et al., 2013). En este contexto, los métodos más utilizados son los modelos geográficamente ponderados (GW, son sus siglas en inglés) desarrollados por Fotheringham et al. (2002). En particular, Harris et al. (2011) implementó el GWPCA, una extensión de PCA para considerar la autocorrelación y la heterogeneidad espacial en la estructura de los datos, es decir contemplar situaciones donde un modelo global no resulta adecuado para describir los datos espaciales (Lloyd, C. 2010; Harris, P., Brunsdon, C. y Charlton, M., 2011). GWPCA supone que la estructura de co-variación de los datos puede variar espacialmente. Siguiendo a Gollini et al. (2015), si la localización espacial i tiene coordenadas (u,v), la matriz de covarianzas local es:

$$
\Sigma(u, v)=X^{T} W(u, v) X
$$

donde $X$ es la matriz $(n x p)$ de datos y $W(u, v)$ es una matriz diagonal de pesos que depende de la localización $(u, v)$. Esta matriz de pesos puede ser generada usando una función kernel. En nuestro caso de estudio, usamos la función bi-square:

$$
w_{i j}=\left(1-\left(d_{i j} / b\right)^{2}\right)^{2} \text { if } d_{i j} \leq b ; \quad w_{i j}=0 \text { para otro valor }
$$

donde los pesos $\left[\mathrm{w}_{\mathrm{ij}}\right]$ dependen de las distancias entre las localizaciones i y j $\left[\mathrm{d}_{\mathrm{ij}}\right]$ y de la bandwwidth [b], la cual determina el tamaño de la vecindad. Esta medida (constante o

${ }^{3}$ MULTISPATI permite estudiar las relaciones entre las variables (análisis co-variabilidad) y la estructura espacial (autocorrelación) simultáneamente. 
variable) puede ser definida exógenamente (por el usuario) o determinada automáticamente con algún algoritmo de optimización. Para encontrar las componentes principales locales, la descomposición de la matriz de covarianzas local provee los autovalores y autovectores locales:

$$
L\left(u_{i}, v_{i}\right) V\left(u_{i}, v_{i}\right) L\left(u_{i}, v_{i}\right)^{T}=\Sigma\left(u_{i}, v_{i}\right)
$$

donde $L\left(u_{i}, v_{i}\right)$ es una matriz de autovectores local y $V\left(u_{i}, v_{i}\right)$ es una matriz de autovalores local. Finalmente, el GWPCA con $p$ variables genera para cada localización espacial $p$ componentes principales locales y $p$ autovalores con sus autovectores asociados.

Para testear la presencia de heterogeneidad espacial y justificar la aplicación de GWPCA Harris et al. propusieron un test (aleatorizado) Monte Carlo que evalúa si los autovalores locales varían significativamente a través del espacio geográfico. Siguiendo a Nardo et al. (2008), el proceso de construcción del IB se plantea como primer paso la definición de un marco conceptual adecuado, que permitirá definir las dimensiones del fenómeno a estudiar. GMM (2011) proponen tres dimensiones de análisis: Magnitudes Agregadas, Disponibilidad y Cobertura Geográfica y Acceso y Utilización.

Definido el marco conceptual, se seleccionaron los indicadores parciales adecuados para medir cada una de las dimensiones de análisis. Si bien se consideraron los indicadores propuestos por el GMM para la construcción del Indicador Único de Bancarización MERCOSUR, la selección estuvo limitada por la posibilidad de ser calculados regularmente, a partir de información confiable, de acuerdo a estándares y que se encuentren disponibles para el público en general. La Tabla 1 detalla los indicadores parciales utilizados por dimensión de análisis.

Tabla 1. Indicadores Parciales de Bancarización

\begin{tabular}{|c|c|c|}
\hline $\begin{array}{l}\text { Dimensión de } \\
\text { Análisis }\end{array}$ & Indicador & Notación \\
\hline \multirow{2}{*}{$\begin{array}{l}\text { Magnitudes } \\
\text { Agregadas }\end{array}$} & Saldo de Depósitos al sector privado/Población total & [DEPPOB] \\
\hline & Saldo de Préstamos al sector privado/Población total & [PREPOB] \\
\hline \multirow{4}{*}{$\begin{array}{c}\text { Disponibilidad y } \\
\text { Cobertura } \\
\text { Geográfica }\end{array}$} & Nro. de Sucursales Bancarias cada 1.000 km2 & [SUCKM2] \\
\hline & Número de Cajeros Automáticos cada 1.000 km2 & [CAJKM2] \\
\hline & Nro. de Sucursales Bancarias cada 100.000 habitantes & {$[S \cup C P O B]$} \\
\hline & Nro. de Cajeros Automáticos cada 100.000 habitantes & [CAJPOB] \\
\hline \multirow{2}{*}{$\begin{array}{l}\text { Utilización y } \\
\text { Acceso }\end{array}$} & $\begin{array}{l}\text { Proporción de Población con Cobertura de Servicios } \\
\qquad \text { Bancarios }^{4}\end{array}$ & {$[\mathrm{CO}$} \\
\hline & $\begin{array}{l}\text { Proporción de localidades del departamento que cuenta } \\
\text { con infraestructura bancaria }\end{array}$ & [COBLOC] \\
\hline
\end{tabular}

Fuente: Elaboración propia.

Para el cálculo de los indicadores parciales se utilizó información provista por el BCRA correspondiente a diciembre de 2010 (BCRA, 2010a,b). Los datos de la población se obtuvieron a partir del Censo Nacional de Población, Hogares y Viviendas 2010 (INDEC,

4 Proporción de la población que vive en localidades que cuentan con infraestructura bancaria (presencia de sucursal y/o cajero). 
2010). Si bien existen datos más actualizados sobre el sistema financiero, los últimos datos disponibles referidos a Población, tanto a nivel de localidad como departamental, corresponden al año 2010.

Los datos fueron estandarizados permitiendo que la información disponible pueda ser agregada de manera comparable. En este trabajo, se utiliza GWPCA. Es decir, para cada localización espacial $\left(\mathrm{u}_{\mathrm{i}}, \mathrm{v}_{\mathrm{i}}\right)$, el índice global se define a partir de la agregación de los valores de los indicadores parciales seleccionados. ${ }^{5}$

$$
\begin{gathered}
\operatorname{Ind}_{i}^{\left(u_{i}, v_{i}\right)}=\sum_{j=1}^{p} \alpha_{j}^{\left(u_{i}, v_{i}\right)} \cdot Z_{i j} \\
\alpha_{j}^{\left(u_{i}, v_{i}\right)}=\frac{\sum_{k=1}^{p} a_{j k}^{\left(u_{i}, v_{i}\right)} \cdot \lambda_{k}^{\left(u_{i}, v_{i}\right)}}{\sum_{j=1}^{p} \sum_{k=1}^{p} a_{j k}^{\left(u_{i}, v_{i}\right)} \cdot \lambda_{k}^{\left(u_{i}, v_{i}\right)}}
\end{gathered}
$$

donde $\operatorname{Ind} d_{i}^{\left(u_{i}, v_{i}\right)}$ es el valor del indicador para la i-ésima unidad en la localización $\left(u_{i}, v_{i}\right)$, $\alpha_{j}^{\left(u_{i}, v_{i}\right)}$ es el peso relativo en la localización $\left(u_{i}, v_{i}\right)$ del j-ésimo indicador parcial, $Z_{i j}$ es el valor estandarizado del j-ésimo indicador parcial para la i-ésima unidad, $\lambda_{k}^{\left(u_{i}, v_{i}\right)}$ es el autovalor asociado a la k-ésima $C P$ en la localización $\left(u_{i}, v_{i}\right)$ y $a_{j k}^{\left(u_{i}, v_{i}\right)}$ es el factor de peso correspondiente al j-ésimo indicador parcial en la k-ésima $C P$ en la localización $\left(u_{i}, v_{i}\right)$. Finalmente se decidió transformar el valor del indicador a una escala [0-100] para facilitar su interpretación. ${ }^{6}$

$$
I B_{i}=\frac{\operatorname{Ind} d_{i}-\min \left(\operatorname{Ind}_{i}\right)}{\operatorname{máx}\left(\operatorname{Ind}_{i}\right)-\min \left(\operatorname{Ind}_{i}\right)} \cdot 100
$$

Para realizar el procesamiento estadístico se utilizó el software libre R (www.r-project.org), siendo empleado el paquete GWmodel (Gollini et al., 2015).

\section{RESULTADOS}

García (2018), muestra la presencia de autocorrelación espacial significativa en los indicadores parciales utilizados en esta aplicación empírica, confirmando la existencia de patrones espaciales subyacentes en los datos, lo que podría afectar la estructura de covariación reflejada por un análisis multivariado clásico. Esto motivó la aplicación de la metodología GWPCA. Para la construcción de las $(n=62)$ matrices diagonales de pesos geográficos $W(u, v)$ se consideraron distancias euclídeas, una función kernel bi-square y una bandwidth adaptativa que se encontró automáticamente usando cross-validation approach (Harris et al., 2011). Para justificar el uso de GWPCA, se utilizó un test (aleatorizado) Monte Carlo sugerido por Harris op. cit., el que permitió detectar la presencia de heterogeneidad espacial marginalmente significativa.

Siguiendo la sugerencia de Cámara y Tuesta (2014) se incorporaron en el cálculo del índice todas las componentes ya que el objetivo final no era reducir la dimensionalidad

${ }^{5} \mathrm{El}$ uso de los autovalores asociados a cada CP como ponderación garantiza la mayor incidencia o importancia en el indicador global de aquellas CPs que explican una mayor porción de la variabilidad total de los datos.

${ }^{6}$ El rango del IB es $[0,100]$ donde el 0 indica el menor nivel de bancarización y 100 el mayor. 
de los datos sino mejorar la precisión en la medición. De acuerdo a lo establecido en la sección anterior se definió para cada localización espacial el índice global a partir de la agregación de los valores de los indicadores parciales propuestos. La Figura 1 muestra la distribución espacial del IB departamental para la Región Centro para el año 2010 considerando los siguientes niveles de bancarización: Bajo $[0,20)$ Medio-Bajo [20,40), Medio $[40,60)$, Medio-Alto $[60,80)$ y Alto $[80,100]$.

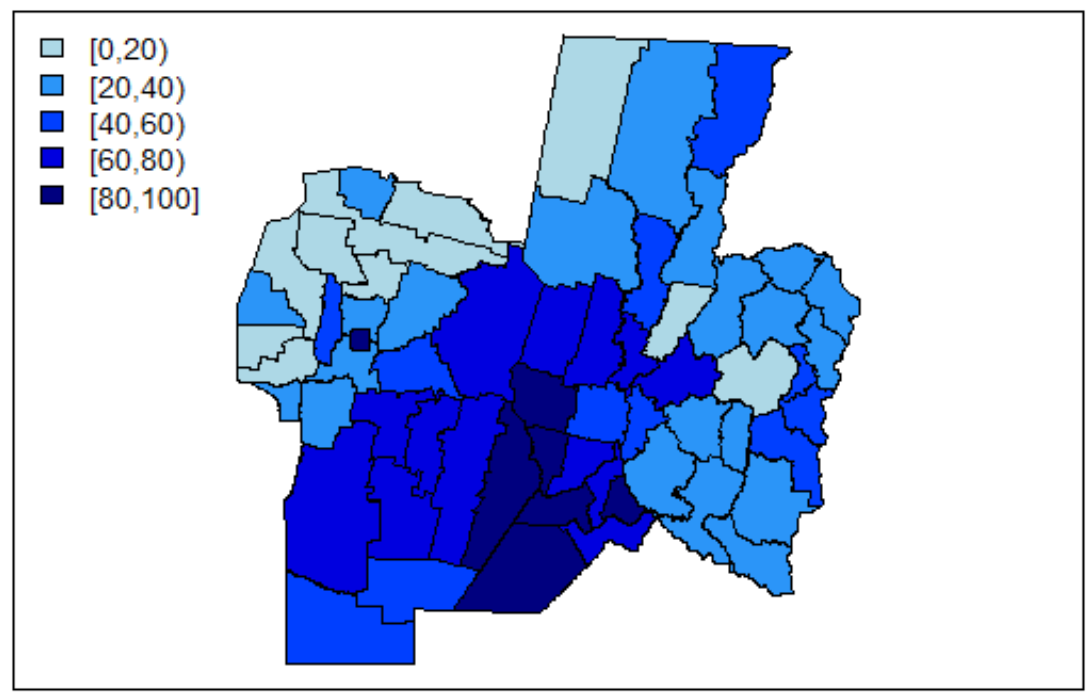

Figura 1. Región Centro: Índice de Bancarización (GWPCA). Año 2010

El gráfico sugiere un mayor nivel de bancarización en la provincia de Santa Fe. La mayoría de los departamentos poseen niveles Medio-Alto y Alto, perteneciendo sólo 5 de los 19 departamentos al nivel Bajo y Medio-Bajo. Sigue en importancia la provincia de Córdoba, pero con un comportamiento más heterogéneo. Esta situación contrasta con el comportamiento exhibido por la provincia de Entre Ríos, que aunque presenta un nivel de bancarización menor, exhibe un comportamiento más homogéneo.

Tabla 2. Índice de Bancarización

\begin{tabular}{llccc}
\hline & \multicolumn{2}{c}{ GWPCA } & \multicolumn{2}{c}{ MULTISPATI } \\
\hline Provincia & Media & $\begin{array}{c}\text { Coeficiente de } \\
\text { Variación (\%) }\end{array}$ & Media & $\begin{array}{c}\text { Coeficiente de } \\
\text { Variación (\%) }\end{array}$ \\
\hline Córdoba & 43,3 & 60,2 & 43,7 & 60,4 \\
Entre Ríos & 34,6 & 32,1 & 37,8 & 29,2 \\
Santa Fe & 58,2 & 45,2 & 60,8 & 39,5 \\
\hline Total & 45,5 & 53,9 & 47,3 & 50,7 \\
\hline
\end{tabular}

Fuente: Elaboración propia.

Esta información puede complementarse con la Tabla 2 que exhibe algunas medidas descriptivas calculadas para el IB obtenido a partir de GWPCA para la región y discriminado por provincia, la que permite confirmar el ordenamiento realizado anteriormente. Comparativamente se muestran también los resultados aplicando 
MULTISPATI. ${ }^{7}$ Si bien el ordenamiento no cambia, las diferencias detectadas obedecen a que esta última metodología ignora la presencia de heterogeneidad espacial.

CONCLUSIONES

Entender el estado actual del proceso de bancarización —o, en otros términos, de -inclusión financiera- de los agentes económicos resulta una cuestión importante, debido al reconocimiento que se le ha dado al sector financiero como elemento que contribuye al desarrollo económico y social. La bancarización resulta importante en tanto constituye una medida del grado de desarrollo del sistema financiero de un país, de la disponibilidad de servicios financieros para la población y las empresas y del nivel de acceso y utilización de los servicios financieros por parte de los distintos agentes económicos. En este sentido, resulta clave una medición adecuada del proceso de bancarización.

Bajo esta premisa, este trabajo propone la construcción de un índice de bancarización para la región Centro de la Argentina para el año 2010 considerando tres dimensiones claves de la bancarización: Magnitudes Agregadas, Disponibilidad y Cobertura Geográfica y Acceso y Utilización. Este índice permite profundizar la comprensión del fenómeno de la bancarización ya que sintetiza de manera sencilla la información proporcionada por los indicadores parciales en una medida sintética y global incorporando la dimensión espacial de los datos. Adicionalmente, resulta un aporte valioso para el diseño de medidas de política apropiadas que promuevan una mejor bancarización.

El uso de GWPCA (Harris et al., 2011) resulta un aporte metodológico significativo, en tanto incorpora la heterogeneidad espacial de los datos en el proceso de construcción del IB. Esta medición, global y sintética, representa la principal fortaleza y aporte del índice propuesto, constituyendo una mejora en relación al presentado en García (2017, 2018) donde se aplica MULTISPATI (Dray et al., 2011), ya que esta metodología supone que los patrones espaciales subyacentes en los datos no varían en todo el espacio geográfico. Así, el índice construido destaca el mayor nivel de bancarización de la provincia de Santa Fe. Completan el ordenamiento la provincia de Córdoba, exhibiendo un menor nivel aunque más heterogéneo, el cual contrasta con el de la provincia de Entre Ríos, la que presenta un bajo nivel de bancarización, aunque más homogéneo.

Este índice debería considerarse como una aproximación nobel a la medición de la bancarización regional, pese a las limitaciones que presenta, vinculadas tanto a la imposibilidad de contar con datos más actualizados como indicadores parciales que midan en forma más adecuada las dimensiones de análisis de la bancarización.

\section{REFERENCIAS}

Amidzic, G., Massara, A., Mialou, A. (2014). Assessing countries' financial inclusion standing: a new composite index. Working Paper n¹4/36, International Monetary Fund (IMF). Recuperado de: https://www.imf.org/external/pubs/ft/wp/2014/wp1436.pdf

${ }^{7}$ Para mayores detalles puede consultarse el trabajo de García (2018). 
BANCO CENTRAL DE LA REPÚBLICA ARGENTINA (2010a). Información de Entidades Financieras. Superintendencia de Entidades Financieras y Cambiarias.

BANCO CENTRAL DE LA REPÚBLICA ARGENTINA (2010b). Disponibilidades, Préstamos y Depósitos clasificados según la ubicación geográfica de la casa o sucursal de la entidad financiera.

BANCO CENTRAL DE LA REPÚBLICA ARGENTINA (2015). Mi diccionario financiero. Recuperado de http://www.bancocentraleduca.bcra.gov.ar/PDFs/Diccionario_Financiero_Jovenes. pdf

Camara, N., Tuesta, D. (2014). Measuring Financial Inclusion: a multidimensional index. BBVA Research, Working Paper $N^{\circ}$ 14/26. Recuperado de: https://www.bbvaresearch.com/wpcontent/uploads/2014/09/WP14-26_Financial-Inclusion2.pdf

Chakravarty, S.; Pal, R. (2013): Measuring Financial Inclusion: an axiomatic approach. Journal of Policy modeling, 35(5), 813-837. Recuperado de: https://ideas.repec.org/p/ind/igiwpp/201003.html

Demsar, U., Harris, P., Brunsdon, C., Fotheringham, A.S., Mcloone, S. (2013): Principal Component Analysis on Spatial Data: An Overview. Annals of the Association of American Geographers, 103(1), 106-28. https://doi.org/10.1080/00045608.2012.689236

Domínguez Serrano, M., Blancas Peral, F.J., Guerrero Casas, F.M., Gonzalez Lozano, M. (2011). Una revisión crítica para la construcción de indicadores sintéticos. Revista de Métodos Cuantitativos para la Economía y la Empresa, 11, 41-70. Recuperado de: https://www.upo.es/revistas/index.php/RevMetCuant/article/view/2094

Dray, S., Jombart, T. (2011). Revisiting Guerry's data: introducing spatial constraints in multivariate Analysis. The Annals of Applied Statistics, 5(4), 2278-2299. https://www.jstor.org/stable/23069330

Fotheringham, A.S., Brunsdon, C., Charlton, M. (2002): Geographically Weighted Regression: The Analysis of Spatially Varying Relationships. John Wiley \& Sons.

García, F. (2017). Un índice único de bancarización con datos georreferenciados con una aplicación para la Argentina. Ecos de Economía, 21(45), 24-38. https://doi.org/10.17230/ecos.2017.45.2

García, F. (2018). ¿Es posible un índice de bancarización en Argentina? Una aplicación espacial para Córdoba, Entre Ríos y Santa Fe. Estudios Económicos, 35(70), 57-77. Recuperado de: https://dialnet.unirioja.es/servlet/articulo? codigo $=7390266$

Gollini, I., Lu, B., Charlton, M.; Brunsdon, C., Harris, P. (2015). GWmodel: an R Package for exploring Spatial Heterogeneity using Geographically Weighted Models. Journal of Statistical Software, 63(17), 1-50.

GRUPO DE MONITOREO MACROECONÓMICO (2011): Indicadores de bancarización. Buenos Aires. Recuperado de https://www.gmm-mercosur.org

Gupte, R., Venkataramani, B., Gupta, D. (2012). Computation of financial inclusion index for India. Procedia - Social and Behavioral Sciences, 37, 133-149. https://doi.org/10.1016/j.sbspro.2012.03.281

Harris, P., Brunsdon, C., Charlton, M. (2011). Geographically weighted principal components analysis. International Journal of Geographical Information Science, 25(10), 1717-1736. https://doi.org/10.1080/13658816.2011.554838

Hasan, R., Islam, E. (2016). Financial Inclusion Index at district levels in Bangladesh: a distance-based approach. Bangladesh Bank, Working Paper Series No 1603. Recuperado de https://ideas.repec.org/p/pra/mprapa/71344.html

INSTITUTO NACIONAL DE ESTADÍSTICAS Y CENSOS (2010). Censo Nacional de Población, Hogares y Viviendas 2010, procesado con Redatam+SP. Recuperado de http://www.indec.gov.ar 
Lloyd, C. D. (2010). Analysing population characteristics using geographically weighted principal components analysis: a case study of Northern Ireland in 2001. Computers, Environment and Urban Systems, 34(5), 389-399. https://doi.org/10.1016/j.compenvurbsys.2010.02.005

Mishra, R. N., Verma, P.; Bose, S. (2014). Operationalising financial inclusion Index as a policy lever: Uttar Pradesh (in India) - A Case Study. Journal of Mathematics and Statistical Science, 2015, 149165.

Moran, P. (1950). Notes on Continuous Stochastic Phenomena. Biometrika, 37(1), 17-23. https://doi.org/10.2307/2332142

Morales, L., Yañez, A. (2006). La bancarización en Chile, concepto y medición. Superintendencia de Bancos e Instituciones Financieras de Chile, Serie Técnica de Estudios. Recuperado de: http://www.cmfchile.cl/portal/publicaciones/610/articles-40031_doc_pdf.pdf

Nardo, M., Saisana, M., Saltelli, A., Tarantola, S., Hoffmann, A., Giovannini, E. (2008). Handbook on Constructing Composite Indicators: Methodology and User Guide. Paris: OECD Publishing. Recuperado

https://www.oecd.org/els/soc/handbookonconstructingcompositeindicatorsmethodologyandus erguide.htm

Sarma, M. (2008). Index of financial inclusion. Working Paper № 215, New Delhi: Indian Council for Research on International Economics Relations.

Sarma, M. (2012). Index of Financial Inclusion - A measure of financial sector inclusiveness. Berlin Working Papers on Money, Trade, Finance and Development, Nº7

Sethy, S. K. (2016). Developing a financial inclusion index and inclusive growth in India. Theoretical and applied economics, 2(607), 187-206. Recuperado de: http://www.ectap.ro/developing-afinancial-inclusion-indexand-inclusive-growth-in-india-susanta-kumar-sethy/a1191/

Zulaica Piñeyro, C. M. (2013). Financial Inclusion index: proposal of a multidimensional measure for México. Revista Mexicana de Economía y Finanzas, 8(2), 157-180. https://doi.org/10.21919/remef.v8i2.46 